\title{
Urinary N-Acetyl-Beta-D-Glucosaminidase Activity in Rat Experimental Ischemic and Toxic Models of Acute Kidney Injury
}

\author{
Andrei Răzvan CODEA ${ }^{1}$, Mircea MIRCEAN ${ }^{1 *}$, Sidonia Alina BOGDAN ${ }^{2}$, Andras Laszlo NAGY2, Alexandra \\ BIRIȘ ${ }^{2}$, Orsolya SARPATAKI ${ }^{2}$, Ionuț IONUȚ ${ }^{1}$, Vlad LUCA $^{2}$, Cristian POPOVICI ${ }^{1}$, Ileana BOGDAN, ${ }^{3}$ Aurora \\ Livia URSACHE, ${ }^{2}$ Liviu Ioan OANA ${ }^{2}$. \\ ${ }^{1}$ Department of Clinical Sciences, Faculty of Veterinary Medicine, University of Agricultural Sciences \\ and Veterinary Medicine, Cluj-Napoca, Romania. \\ ${ }^{2}$ Department of Preclinical Sciences, Faculty of Veterinary Medicine, University of Agricultural Sciences \\ and Veterinary Medicine, Cluj-Napoca, Romania. \\ ${ }^{3}$ Department of Technical and Soil Sciences, Faculty of Agriculture, University of Agricultural Sciences \\ and Veterinary Medicine, Cluj-Napoca, Romania \\ *corresponding author: mircea.mircean@usamvcluj.ro
}

Bulletin UASVM Veterinary Medicine 74(1) / 2017,

Print ISSN 1843-5270; Electronic ISSN 1843-5378

DOI:10.15835/buasvmcn-vm: 12618

\begin{abstract}
The identification of a suitable prevention method which facilitates limiting the deleterious effects of acute kidney injuries is highly required. In order to identify a proper treatment for acute kidney injuries, a suitable experimental model that replicates the structural, metabolic and inflammatory lesions that occur in the natural acute injured kidney is highly necessary. Intense urinary NAG activity can be found in a variety of renal disease such as toxic nephropathies, ischemic renal injury following cardiac surgery or renal transplantation but also in glomerular disease especially in diabetic nephropathy. Rises in urinary NAG enzyme activity strongly suggests tubular cell damage and support NAG enzyme as a biomarker of renal tubular injury. The aim of this paper is to obtain a stable in vivo acute kidney injury experimental model, in Wistar, rats and to evaluate the urinary activity of N-acetyl- $\beta$-D-glucosaminidase (NAG) enzyme, blood levels of urea and creatinine and microstructural renal alterations induced by ischemia/reperfusion injury respectively gentamicin nephrotoxicity. For this purpose we have used a rat experimental model. Adult male Wistar rats weighing 250-300 g were randomly divided into 3 groups with 8 rats in each group. Group 1 served as a model for the renal ischemia/reperfusion injury experiment, group 2 served for toxic kidney injury experimental model and group 3 served as control group. All individuals in both groups 1 and 2 presented marked elevations in blood urea and creatinine at the moment of euthanasia (day 3 for group 1 and day 9 for group 2) compared to the control group where biochemical values remained within normal limits. Urine analysis of both group 1 and 2 showed marked urinary NAG index activity which suggests acute tubular injury, suggestion confirmed by histological evaluation of the renal parenchyma sampled from this subjects
\end{abstract}

Keywords: $N$-Acetyl- $\beta$-D-Glucosaminidase, $N A G$, acute kidney injury, ischemia/reperfusion, gentamicin, nephrotoxicity.

\section{INTRODUCTION}

Ischemia/reperfusion injury as a consequence of renal surgery, renal transplantation, shock, cardiac arrest renal together with various toxic injuries represents one of the major causes of acute kidney injury
(Gasanov et al., 2011). Although extensive research performed in the last decades have substantially improved the knowledge of the mechanisms and pathological pathways involved in the development of renal ischemia/reperfusion injury and nephrotoxic 

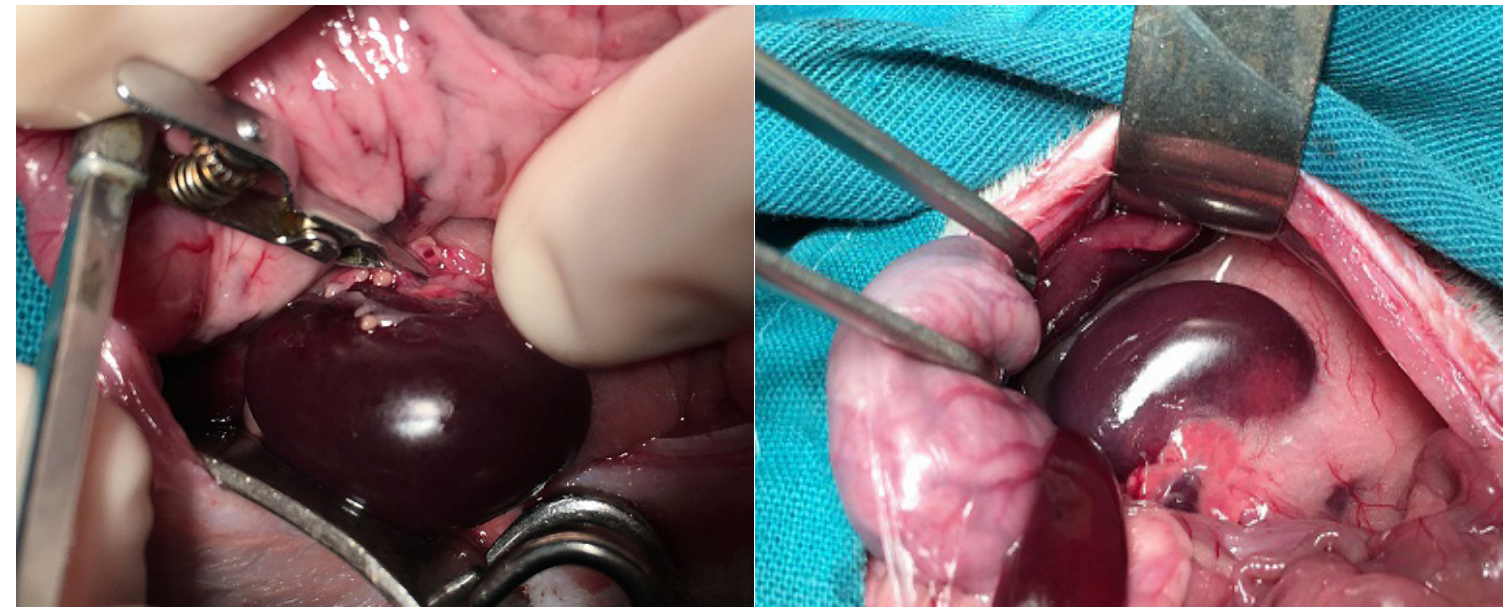

Fig. 1 Isotation of the right kidney

injuries, an effective treatment for such lesions is still unavailable. Therefore, the identification of a suitable method to prevent and minimize the clinical effects of such injuries is highly required. In order to identify a proper treatment for acute kidney injuries, a stable experimental model that replicates the structural, metabolic and inflammatory lesions that occur in the natural acute injured kidney is mandatory.

$\mathrm{N}$-Acetyl- $\beta$-D-Glucosaminidase (NAG) is a lisosomal enzyme located especially inside the epithelial cells of the renal proximal convoluted tubules. Rises in urinary NAG enzyme activity strongly suggests tubular cell damage and support NAG as a biomarker of renal tubular injury. During the course of renal disease, urinary NAG values remain permanently elevated. Intense urinary NAG activity can be found in a variety of renal disease such as toxic nephropathies, ischemic renal injury following cardiac surgery or renal transplantation but also in glomerular disease especially in diabetic nephropathy (Lerma et al., 2014, Obermuller et al., 2014).

Our aim was to evaluate the urinary activity of N-Acetyl- $\beta$-D-Glucosaminidase when ischemia/ reperfusion injury or gentamicine toxicity is a contributing factor.

\section{MATERIALS AND METHODS}

Twenty-one healthy adult male Wistar rats weighing 250-300 g were selected for this study. All animals were cared for according to the experimental medicine protocols of University of Agricultural Sciences and Veterinary Medicine Cluj-Napoca. The animals were housed in an environmentally controlled animal house, in polypropylene rodent cages, at a temperature of $22 \pm 1^{\circ} \mathrm{C}$ with a $12 \mathrm{~h}$ light/dark cycle and were allowed free access to drinking water and a standard granulated rodent laboratory rat diet.
Fig. 2 Right renal pedicle clamping

The rats were randomly divided into 3 groups with 8 rats in each group. Group 1 served for the renal ischemia/reperfusion injury experiment, group 2 served for toxic kidney injury experiment and group 3 served as control group. Rats in group 1 were anesthetized with intramuscular injection of Xilazyne (Xilazin Bio® 2\%, Bioveta, Czech Republic) followed by $5 \mathrm{mg} / \mathrm{kg}$ and Ketamine $50 \mathrm{mg} / \mathrm{kg}$ (Ketamidor $\AA$ $100 \mathrm{mg} / \mathrm{ml}$, Richter Pharma, Austria). After surgical preparation of the abdomen a midline celiotomy was performed and bilateral renal pedicles were isolated using minimal blunt dissection. Both renal pedicles were temporary occluded by surgical clamping for 1 hour using atraumatic microvascular clamps followed by reperfusion and abdominal wall closure (fig. 1 and 2 ). The animals were housed for three days in the same conditions. Enrofloxacine $5 \mathrm{mg} / \mathrm{kg} /$ day (Enroxil@ 10\%, KRKA), intramuscular injection was given for post operatory infection prevention 3 days consecutively. Tramadol $20 \mathrm{mg} / \mathrm{kg} /$ day (Tramadol $® 50 \mathrm{mg} / \mathrm{ml}$, KRKA) intramuscular injection was administered for postoperative analgesia 3 days consecutively. All animals were euthanized in the third day after surgery by means of Isoflurane euthanasia chamber. Whole blood was processed in order to measure serum urea and creatinine concentrations. Urinary NAG index activity was evaluated in urine samples obtained by cystotomy immediately after euthanasia. Kidney samples were obtained for histological examination.

Rats in group 2 were intraperitonealy injected with $100 \mathrm{mg} / \mathrm{kg}$ gentamicine sulphate (Gentamicin ${ }^{\circledR} 40 \mathrm{mg}$ / ml KRKA) for 8 consecutive days (Yaman and Balikci, 2009; Whiting and Brown, 1996). All individuals were euthanized in day 9 of the experiment by means of Isoflurane (Anesteran $®$, Rompharm, Romania) euthanasia chamber. Blood samples were collected and serum was obtained for urea and creatinine dosing. 


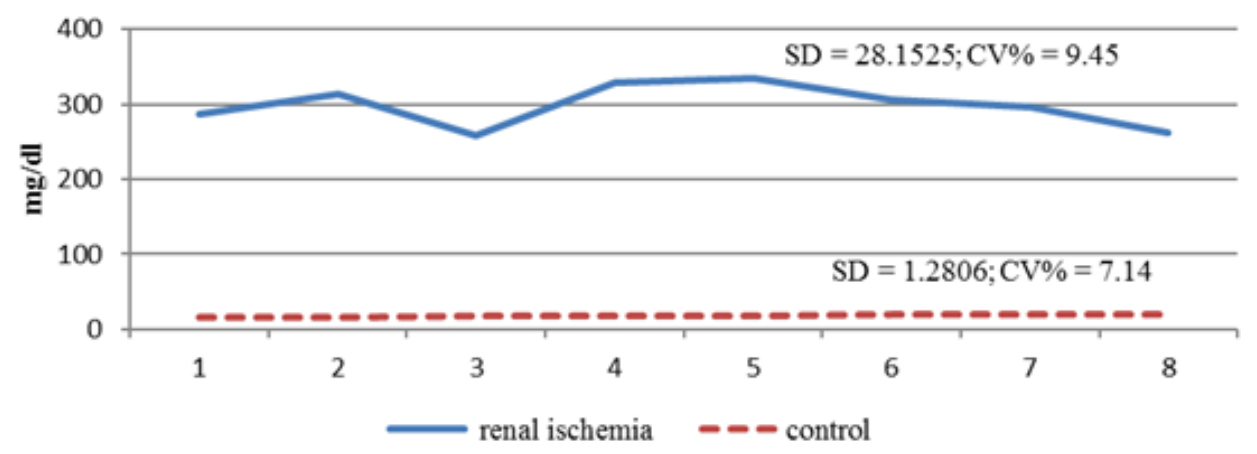

Fig.3. Renal ischemia/reperfusin influence on urea

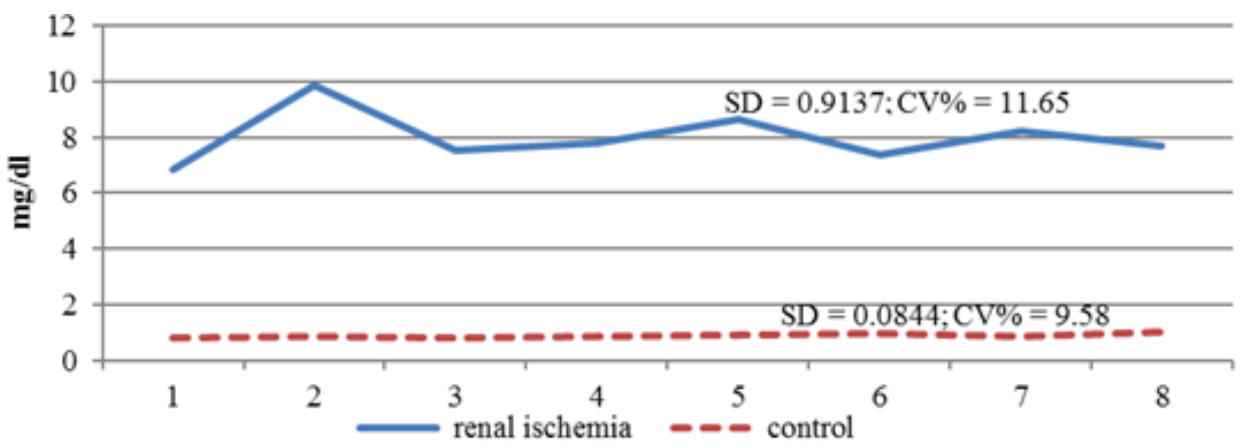

Fig.4. Renal ischemia influence on creatinine

Immediately after euthanasia urine was collected by cistotomy for NAG index activity evaluation. Complete necropsy was performed in all cases, and the kidneys were removed for histological examination.

\section{Biochemical analysis:}

Serum creatinine concentration was measured using a spectrofotometric kinetic method and Jaffe reaction while serum urea concentration was measured using a spectrophotometric kinetic method. In order to determine the Urinary NAG index, first urine samples were centrifuged at $1000 \mathrm{rpm}$ for $5 \mathrm{~min}$ at $4^{\circ} \mathrm{C}$ then the enzymatic activity was measured by a spectrophotometric, cholorimetric method. NAG index was measured using an end-point spectrophotometric reaction whereas the urinary concentration of creatinine was determined by a spectrophotometric, kinetic reaction using the Jaffe method. Urinary NAG index was calculated by the following equation: NAG index $(\mathrm{U} / \mathrm{g})=$ urinary $\mathrm{NAG}$ activity $(\mathrm{U} / \mathrm{l})$ / urinary creatinine concentration (g/l) (Sato et al., 2002).

Statistical analysis: Results for each parameter were processed by average, standard deviation and verified by coefficient of variation. The experimental groups were compared with the control for each parameter.

\section{Histological analysis:}

A Complete necropsy was performed in all cases, and the kidneys were removed for histological analysis. The renal samples were fixed in 10\% neutral buffered formalin and embedded in paraffin wax. Serial sections $(4 \mu \mathrm{m})$ were realized for routine Haematoxylin and Eosin (H\&E) staining. The kidney sections were examined using an Olympus BX 51 microscope and the images were taken with Olympus UC 30 digital camera and processed using an image acquisition and processing program Olympus Stream Basic.

\section{RESULTS AND DISCUSSION}

In both groups all individuals presented marked elevations in blood urea and creatinine concentrations at the moment of euthanasia (day 3 for group 1 and day 9 for group 2). Increased urinary NAG index activity indicates acute tubular injury, which was also confirmed by renal histology analysis. values

Control group urea, creatinine and NAG index

The control group recorded an average of 17.9375 urea in $\mathrm{mg} / \mathrm{dl}$, with a standard deviation (SD) of 1.2806 $\mathrm{mg} / \mathrm{dl}$ (Fig. $3 \& 5$ ). The variation within the group is reduced, the group is homogeneous in terms of this parameter, the coefficient of variation was below $10 \%$ : 


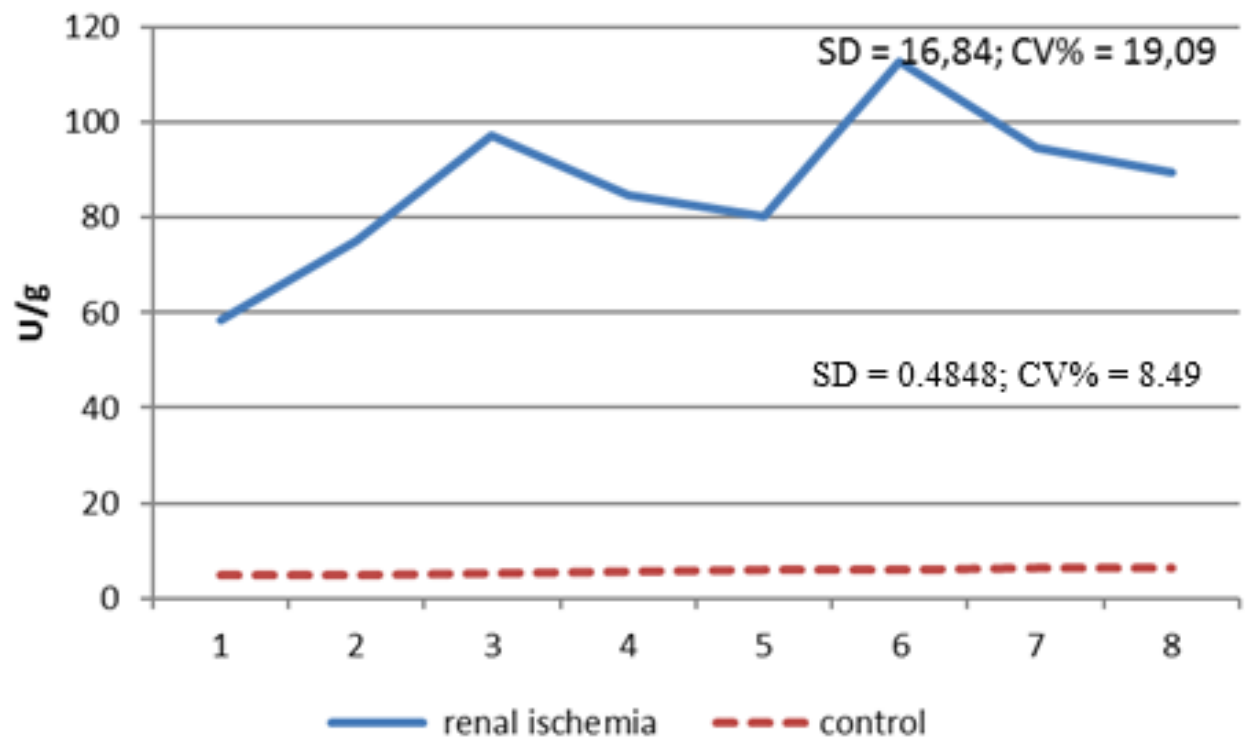

Fig.5. Renal ischemia influence on NAG index

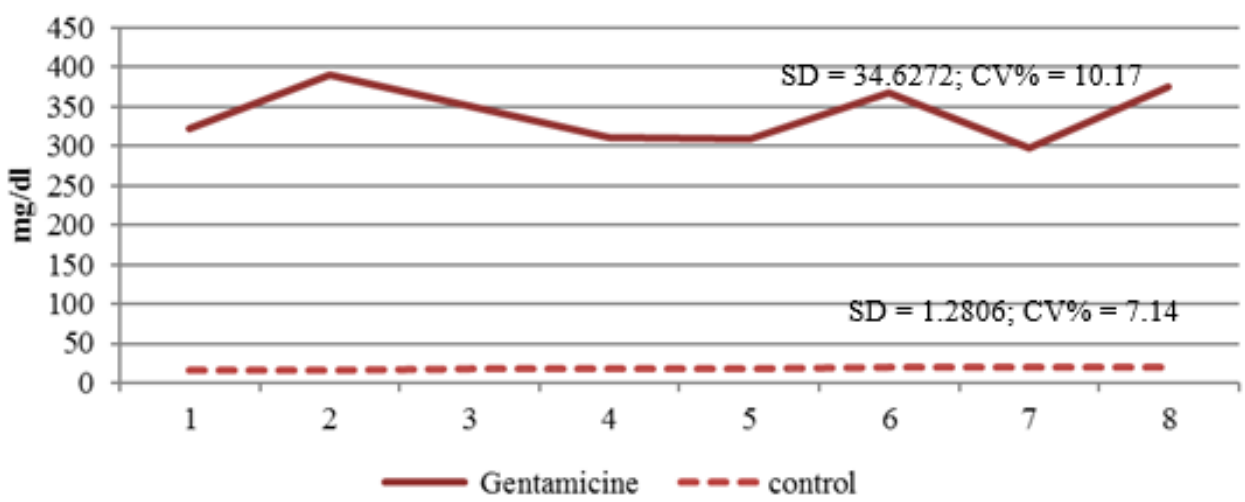

Fig.6. Gentamicine Nephrotoxicity influence on urea

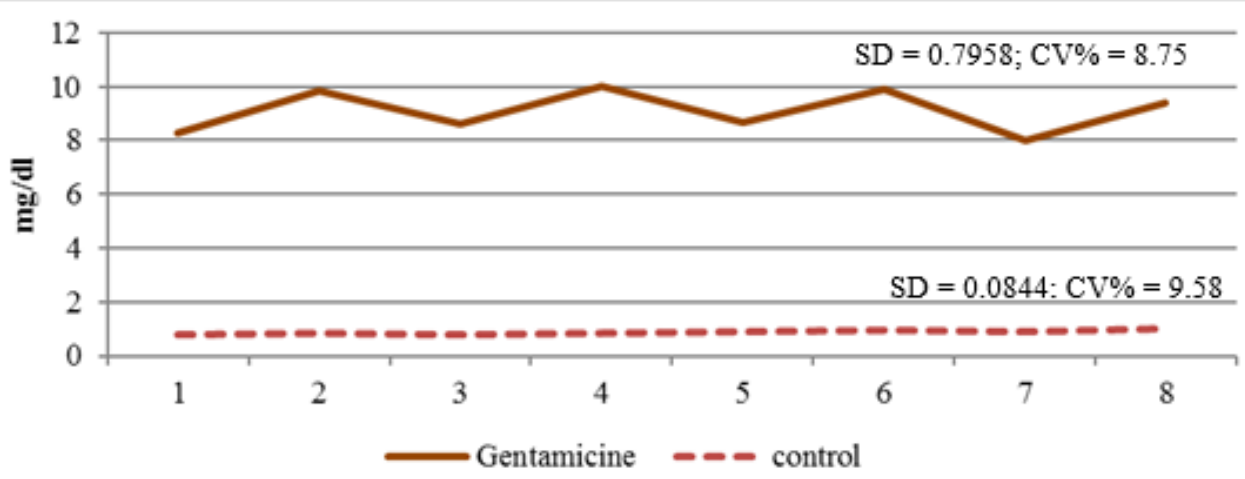

Fig.7. Gentamicine nephrotoxicity influence on creatinine

C.V\% $=7.14$. Creatinine recorded in the control group varies between 0.8 and $1.02 \mathrm{~g} / \mathrm{dL}$, with an average of $0.88125 \mathrm{mg} / \mathrm{dL}$ and a standard deviation of $0.0844 \mathrm{mg} /$
dL. The control group is homogeneous, coefficient of variation of creatinine has value $9.58 \%$ (Figure 4 \& 7). 


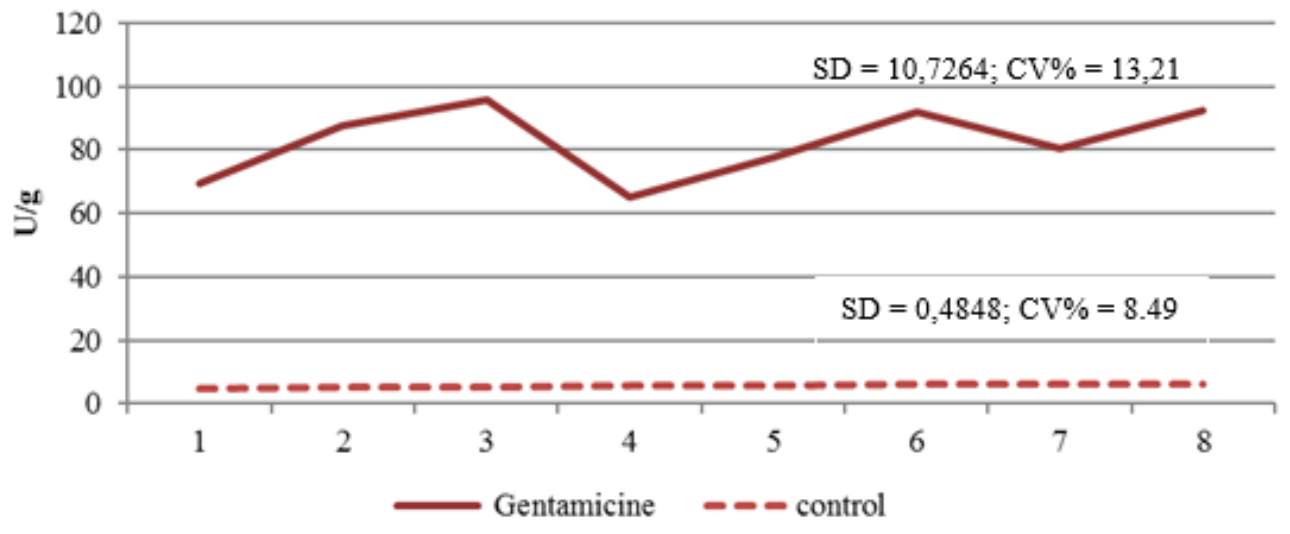

Fig.8. Gentamicine nephrotoxicity influence on NAG Index

NAG index has averaged $5.71125 \mathrm{U} / \mathrm{g}$ in the control group, with a standard deviation of 0.4848 and a low variation within the batch. C.V.\% $\% 8.49$ (Fig. 5 \& 8).

\section{Renal ischemia/reperfusion influence on urea, creatinine and NAG index values}

Following induction of renal ischemia, urea values rised over 15 times, between $258.3 \mathrm{mg} / \mathrm{dl}$ and 334.5 $\mathrm{mg} / \mathrm{dL}$. Average urea value is $298.0625 \mathrm{mg} / \mathrm{dL}$ with a standard deviation of $28.1525 \mathrm{mg} / \mathrm{dL}$. Variation of urea in the renal ischemia/reperfusion rat group is reduced: C.V\% $=9.45$ (Fig. 3)

Renal ischemia/reperfusion injury produces a increase of about 9 times in creatinine concentration so that creatinine values recorded in the group have an average of $7.99875 \mathrm{mg} / \mathrm{dL}$. the mean standard deviation of $0.9137 \mathrm{mg} / \mathrm{dL}$ (Fig. 4).

NAG index increases by an average of 15 due to renal ischemia/reperfusion injury (Fig. 5) NAG index values recorded where within the range of 75.1-112.8 $\mathrm{U} / \mathrm{g}$. Group average is $88.22857 \mathrm{U} / \mathrm{g}$, with a standard deviation of $16.84 \mathrm{U} / \mathrm{g}$ and an average variation values: C.V $=19.09 \%$.

The influence of gentamicine nephrotoxicity on urea, cratinine and NAG index values

Following gentamicin administration urea values rised by nearly 20 times, between $298.6 \mathrm{mg} / \mathrm{dL}$ and $389.7 \mathrm{mg} / \mathrm{dL}$. Urea lot average was $340.55 \mathrm{mg} / \mathrm{dL}$, with a standard deviation of $34.6272 \mathrm{mg} / \mathrm{dL}$. Urea variation in the rat group with gentamicine nephrotoxicity is low to medium: C.V\% = 10.17 (Fig. 6).

Creatinine increases nearly 10 times, the average value for the group of rats with gentamicin nephrotoxicity is $9.095 \mathrm{mg} / \mathrm{dL}$, with a standard deviation of $0.7958 \mathrm{mg} / \mathrm{dL}$ and a low variation: $\mathrm{C} . \mathrm{V}=$ 9.58\% (Fig. 7).

NAG index increases by an average of 14 times due to gentamicine nephrotoxicity (Fig. 8) so that in the gentamicine nephrotoxicity group NAG index values were found to be between 64.9 and $95.7 \mathrm{U} / \mathrm{g}$.
Lot average was $81.225 \mathrm{U} / \mathrm{g}$, with a standard deviation of $10.73 \mathrm{U} / \mathrm{g}$ and a variation in average values: $\mathrm{C} . \mathrm{V}=$ $3.21 \%$.

\section{Histological studies \\ Group 1}

Grossly, areas of congestion and necrosis were observed in the cortex of the kidney. Microscopically, the renal morphological alterations were represented by necrosis and mineralization of the renal tubules and of some glomeruli from the cortex, without any inflammatory infiltrate. The epithelial cells of the proximal and distal convoluted tubules were detached from the basement membrane, with pyknotic nuclei and mineralization. No signs of regeneration were observed (fig. 9).

\section{Group 2}

All animals showed massive tubular necrosis with the presence of granular eosinophilic material in the tubular lumen. In most of the cases even the basement membrane was destroyed. Some tubules were still intact, with granular eosinophilic material within the tubular epithelial cells (hyaline). Hyaline casts were also observed in some sections. No signs of mineralization were present. Slight mononuclear inflammatory infiltrate was observed in all cases (Friedewald et al., 2004) (fig.10).

\section{CONCLUSION}

Our study demonstrates that both induction of warm renal ischemia/reperfusion injury by bilateral renal pedicle surgical occlusion and acute kidney injury by administration of nephrotoxic doses of gentamicin are feasible methods for obtaining a proper in vivo model of acute kidney injury. Both experimental models demonstrate a increase in urinary NAG values which are reflected by the histological alterations induced by renal ischemia/reperfusion respectively gentamicin nephrotoxicity. The renal extent injuries were also 


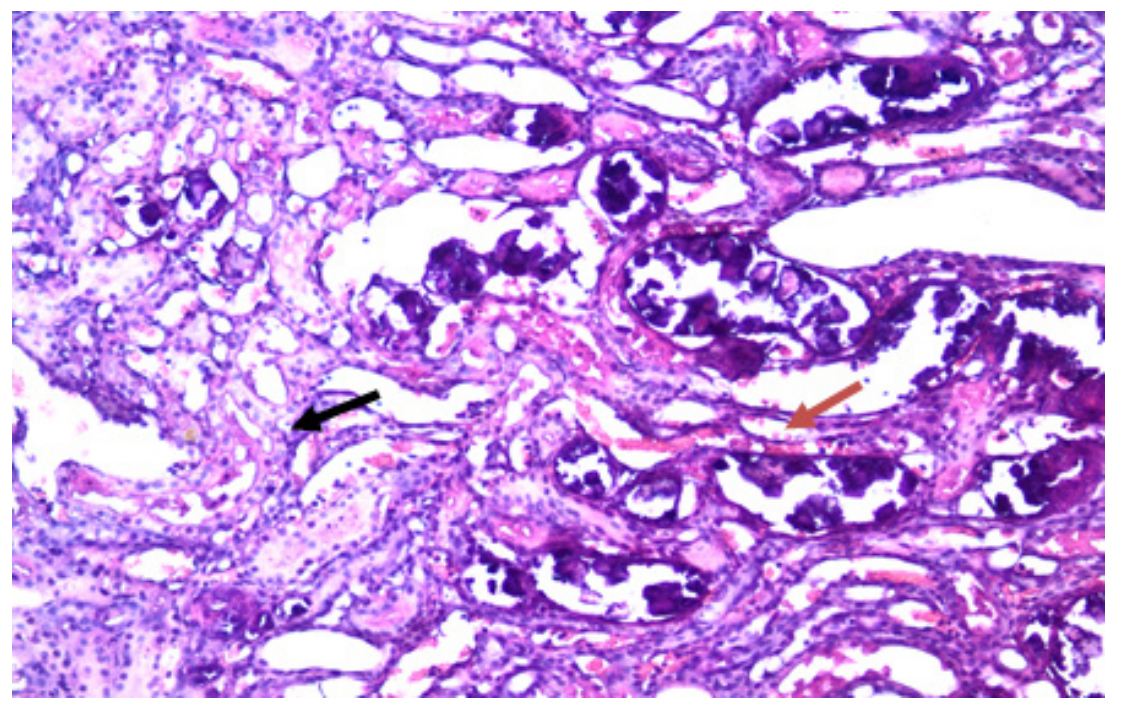

Fig.9. Severe necrosis (black arrow) and mineralization (red arrow) of the renal tubules, no signs of regeneration were observed HEx200.

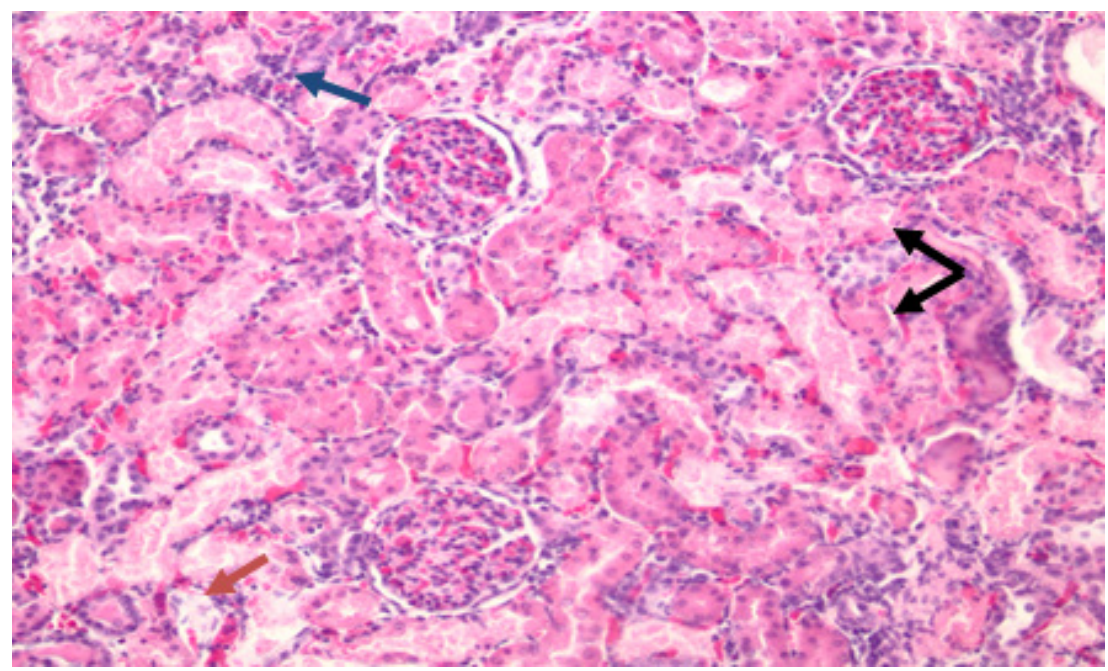

Fig.10. Massive renal tubular necrosis (black arrows), mononuclear inflammatory infiltrate (blue arrow), renal hyalinosis (red arrow) HEx200.

proved by significant elevation of serum creatinine and urea in both experimental groups.

\section{REFERENCES}

1. Friedewald JJ, Rabb H. (2004). Inflammatory cells in ischemic acute renal failure. Kidney International, 66 (2): $486-491$.

2. Gasanov F, Aytac B , Vuruskan H. (2011). The effects of tadanafil on renal ischemia reperfusion injury: an experimental study. Boston J Basic Med Sci, 11(3):158162.

3. Yaman I, Balikci E (2009). Protective effects of nigella sativa against gentamicin-induced nephrotoxicity in rats. Experimental and Toxicologic Pathology 62 (2): 183-190.
4. Lerma VE, Agraharkar M, Kelly B, Tan JA (2014). Novel biomarkers of renal function. http://emedicine.medscape. com/article/1925619-overview\#a1

5. Obermuller N, Geiger H, Weipert C, Urbscha A (2014). Current developments in early diagnosis of acute kidney injury. Int Urol Nephrol 46:1-7.

6. Sato R, Soetam S, Miyazaki M, Syuto B, Sato J, Miyake Y, Yasuda J, Okada K, Naito Y (2002). Clinical availability of urinary $\mathrm{N}$-acetyl- ${ }^{\beta}$-D-Glucosaminidase index in dogs with urinary diseases. J. Vet.Med.Sci, 64 (4):361-365.

7. Whiting PH, Brown PA (1996). The Relationship Between Enzymuria and Kidney Enzyme Activities in Experimental Gentamicin Nephrotoxicity. Journal of Renal failure, 18 (6): 899-909. 